\title{
Hypercalcemia Is of Uncertain Significance in Patients With Advanced Adenocarcinoma of the Prostate
}

\author{
Ghaith Alhatemi, MD; Rafal Alhatemi, MD; Haider Aldiwani, MD; Marwah Hussein, MD; Edi Levi, MD; and Pallavi Jasti, MD \\ Hypercalcemia in the setting of prostate cancer is rare with an uncertain pathophysiology and \\ more research is needed into the role of parathyroid hormone-related peptide as a growth factor \\ and possibly target-directed monoclonal antibody therapies.
}

\author{
Ghaith Alhatemi and \\ Marwah Hussein are \\ Internists and Rafal \\ Alhatemi is a Research \\ Assistant, all at the Detroit \\ Medical Center, Wayne State \\ University in Michigan. \\ Haider Aldiwani is an \\ Internist at Scripps Mercy \\ Hospital Chula Vista in San \\ Diego, California. Edi Levi \\ is a Pathologist and Pallavi \\ Jasti is a Medical \\ Oncologist, both at John \\ Dingell VA Medical Center \\ in Detroit. \\ Correspondence: \\ Ghaith Alhatemi \\ (galhatem@med.wayne.edu)
}

$\mathrm{H}$ ypercalcemia is found when the corrected serum calcium level is $>10.5 \mathrm{mg} / \mathrm{dL} .^{1}$ Its symptoms are not specific and may include polyuria, dehydration, polydipsia, anorexia, nausea and/or vomiting, constipation, and other central nervous system manifestations, including confusion, delirium, cognitive impairment, muscle weakness, psychotic symptoms, and even coma. ${ }^{1,2}$

Hypercalcemia has varied etiologies; however, malignancy-induced hypercalcemia is one of the most common causes. In the US, the most common causes of malignancy-induced hypercalcemia are primary tumors of the lung or breast, multiple myeloma (MM), squamous cell carcinoma of the head or neck, renal cancer, and ovarian cancer. ${ }^{1}$

Men with prostate cancer and bone metastasis have relatively worse prognosis than do patient with no metastasis. ${ }^{3}$ In a recent meta-analysis of patients with bone-involved castration-resistant prostate cancer, the median survival was 21 months. ${ }^{3}$

Hypercalcemia is a rare manifestation of prostate cancer. In a retrospective study conducted between 2009 and 2013 using the Oncology Services Comprehensive Electronic Records (OSCER) warehouse of electronic health records (EHR), the rates of malignancy-induced hypercalcemia were the lowest among patients with prostate cancer, ranging from 1.4 to $2.1 \%$.

We present this case to discuss different pathophysiologic mechanisms leading to hypercalcemia in a patient with prostate cancer with bone metastasis and to study the role of humoral and growth factors in the pathogenesis of the disease.

\section{CASE PRESENTATION}

An African American man aged 69 years presented to the emergency department (ED) with generalized weakness, fatigue, and lower extremities muscle weakness. He reported a 40 -lb weight loss over the past 3 months, intermittent lower back pain, and a 50 pack-year smoking history. A physical examination suggested clinical signs of dehydration.

Laboratory test results indicated hypercalcemia, macrocytic anemia, and thrombocytopenia: calcium $15.8 \mathrm{mg} / \mathrm{dL}$, serum albumin $4.1 \mathrm{mg} / \mathrm{dL}$, alkaline phosphatase $139 \mu / \mathrm{L}$, blood urea nitrogen $55 \mathrm{mg} / \mathrm{dL}$, creatinine $3.4 \mathrm{mg} / \mathrm{dL}$ (baseline 1.4-1.5 mg/dL), hemoglobin $8 \mathrm{~g} / \mathrm{dL}$, mean corpuscular volume $99.6 \mathrm{fL}$, and platelets $100,000 / \mu \mathrm{L}$. The patient was admitted for hypercalcemia. His intact parathyroid hormone (iPTH) was suppressed at $16 \mathrm{pg} / \mathrm{mL}$, phosphorous was $3.8 \mathrm{mg} / \mathrm{dL}$, parathyroid hormone-related peptide $(\mathrm{PTHrP})$ was $<0.74 \mathrm{pmol} / \mathrm{L}$, vitamin $\mathrm{D}$ (25 hydroxy cholecalciferol) was mildly decreased at $17.2 \mathrm{ng} / \mathrm{mL}$, and 1,25 dihydroxy cholecalciferol (calcitriol) was $<5.0$ (normal range $20-79.3 \mathrm{pg} / \mathrm{mL}$ ).

A computed tomography (CT) scan of the chest and abdomen was taken due to the patient's heavy smoking history, an incidentally detected right lung base nodule on chest X-ray, and hypercalcemia. The CT scan showed multiple right middle lobe lung nodules with and without calcifications and calcified right hilar lymph nodes (Figure 1). 

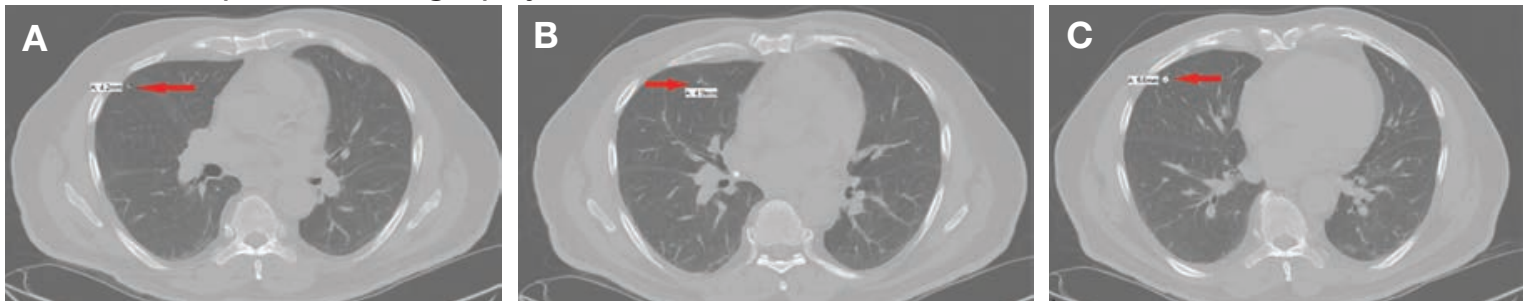

Red arrows point toward nodules. A, A 4-mm noncalcified pulmonary nodule demonstrated in the right middle lobe. B, A 5-mm noncalcified pulmonary nodule shown in the right middle lobe along the fissure. C, A calcified nodule can be seen in the right middle lobe measuring about $6 \mathrm{~mm}$.

\section{FIGURE 2 Histopathology of Bone Marrow Infiltrate With Tumor Cells}
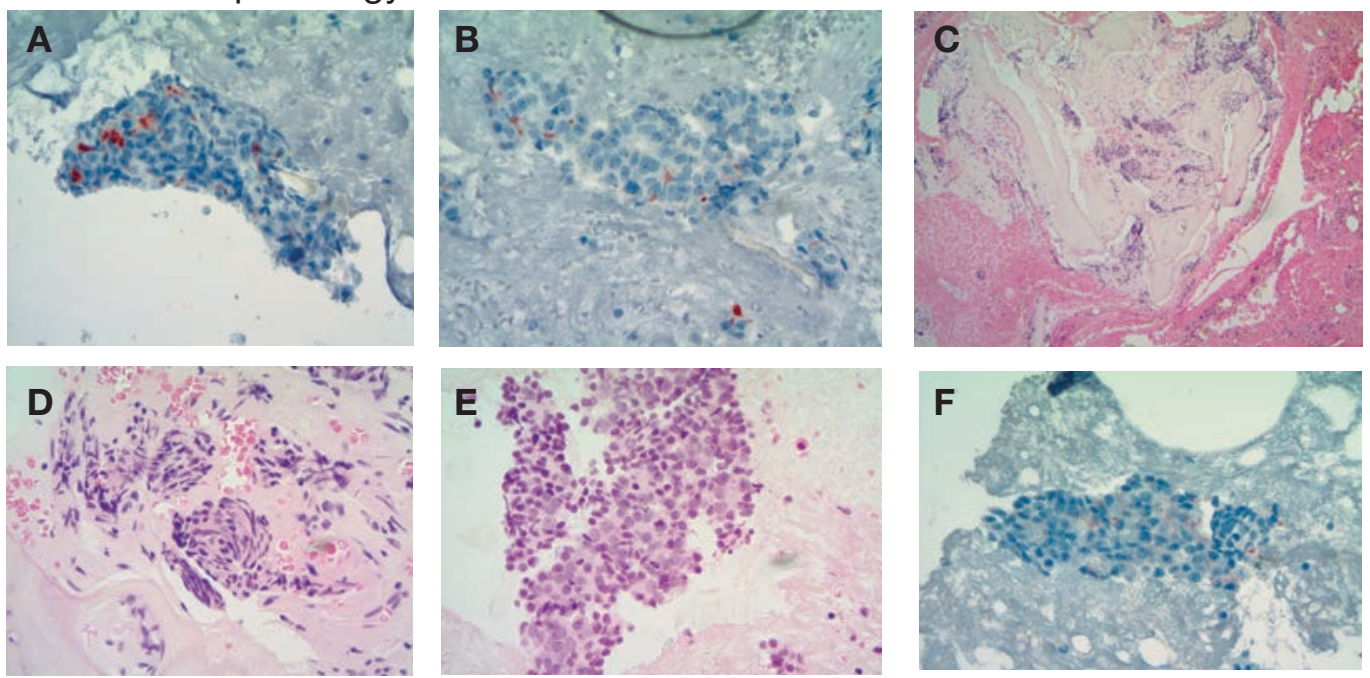

These cells are arranged in solid sheets that are focally cribriforming with glandular spaces; tumor cells are composed of enlarged nuclei with prominent nucleoli, morphologically consistent with prostatic adenocarcinoma. A and B show prostate-specific membrane antigen immunohistochemical staining; C (low power), D, and E (high power) show haemotoxylin and eosin stain; F demonstrates negative neuroendocrine marker chromogranin.

To evaluate the pancytopenia, a bone marrow biopsy was done, which showed that 80 to $90 \%$ of the marrow space was replaced by fibrosis and metastatic malignancy. Trilinear hematopoiesis was not seen (Figure 2 ). The tumor cells were positive for prostate-specific membrane antigen (PSMA) and negative for cytokeratin 7 and 20 (CK7 and CK20). ${ }^{4}$ The former is a membrane protein expressed on prostate tissues, including cancer; the latter is a form of protein used to identify adenocarcinoma of unknown primary origin (CK7 usually found in primary/ metastatic lung adenocarcinoma and CK20 usually in primary and some metastatic diseases of colon adenocarcinoma). ${ }^{5}$ A prostatic specific antigen (PSA) test was markedly elevated: $335.94 \mathrm{ng} / \mathrm{mL}(1.46 \mathrm{ng} / \mathrm{mL}$ on a previous 2011 test).

Metastatic adenocarcinoma of the pros- tate was diagnosed without a prostate biopsy. To determine the extent of bone metastases, a technetium-99m-methylene diphosphonate (MDP) bone scintigraphy demonstrated a superscan with intense foci of increased radiotracer uptake involving the bilateral shoulders, sternoclavicular joints, and sternum with heterogeneous uptake involving bilateral anterior and posterior ribs; cervical, thoracic, and lumbar spines; sacrum, pelvis, and bilateral hips, including the femo$\mathrm{ral} \mathrm{head/neck} \mathrm{and} \mathrm{intertrochanteric} \mathrm{regions.}$ Also noted were several foci of radiotracer uptake involving the mandible and bilateral skull in the region of the temporomandibular joints (Figure 3).

The patient was initially treated with IV isotonic saline, followed by calcitonin and then pamidronate after kidney function improved. His calcium level responded to the 
TABLE Patient Laboratory Results

\begin{tabular}{|c|c|c|c|c|c|c|c|}
\hline Time of Result & $\mathrm{Ca}, \mathrm{mg} / \mathrm{dL}$ & PO4, mg/dL & Alb, g/dL & iPTH, pg/mL & PTHrP, ng/L & GFR, mL/min & Explanation \\
\hline At diagnosis & 15.8 & 3.8 & 4.1 & 16 & $<0.74$ & $\sim 22$ & PTH in-HCa, AKI \\
\hline At discharge & 12.4 & None & 3.8 & None & None & $\sim 52$ & PTH in- $\mathrm{HCa}$ \\
\hline 9-mo postdischarge & 8.6 & 2.9 & 3.3 & 178 & None & $40-45$ & SHPT, CKD \\
\hline 13-mo postdischarge & 8.7 & 4 & 2.7 & 141 & None & $25-30$ & SHPT, worsening CKD \\
\hline $\begin{array}{l}\text { 16-mo postdischarge } \\
\text { (death) }\end{array}$ & 8.2 & None & 3.7 & None & None & $25-30$ & SHPT, CKD \\
\hline
\end{tabular}

Abbreviations: AKI, acute kidney injury; Alb, albumin; Ca, calcium; CKD, chronic kidney disease; GFR, glomerular filtration rate; iPTH, intact parathyroid hormone; PO4, phosphorous; PTHrP, parathyroid hormone-related peptide; PTH in HCa, parathyroid hormone independent hypercalcemia; SHPT, secondary hyperparathyroidism.

therapy, and a plan was made by medical oncology to start androgen deprivation therapy (ADT) prior to discharge.

He was initially treated with bicalutamide, while a luteinizing hormone-releasing hormone agonist (leuprolide) was added 1 week later. Bicalutamide was then discontinued and a combined androgen blockade consisting of leuprolide, ketoconazole, and hydrocortisone was started. This therapy resulted in remission, and PSA declined to $1.73 \mathrm{ng} /$ $\mathrm{mL} 3$ months later. At that time the patient enrolled in a clinical trial with leuprolide and bicalutamide combined therapy. About 6 months after his diagnosis, patient's cancer progressed and became hormone refractory disease. At that time, bicalutamide was discontinued, and his therapy was switched to combined leuprolide and enzalutamide. After 6 months of therapy with enzalutamide, the patient's cancer progressed again. He was later treated with docetaxel chemotherapy but died 16 months after diagnosis.

Follow-up over the course of therapy showed improvement of hypercalcemia at the time of discharge, but 9 months later and toward the time of expiration, our patient developed secondary hyperparathyroidism, with calcium maintained in the normal range, while iPTH was significantly elevated, a finding likely explained by a decline in kidney function and a fall in glomerular filtration rate (Table).

\section{DISCUSSION}

Hypercalcemia in the setting of prostate cancer is a rare complication with an uncertain pathophysiology. ${ }^{6}$ Several mechanisms have been proposed for hypercalcemia of malignancy, these comprise humoral hypercalcemia of malignancy mediated by increased PTHrP; local osteolytic hypercalcemia with secretion of other humoral factors; excess extrarenal activation of vitamin D $(1,25[\mathrm{OH}] 2 \mathrm{D})$; PTH secretion, ectopic or primary; and multiple concurrent etiologies. ${ }^{7}$

PTHrP is the predominant mediator for hypercalcemia of malignancy and is estimated to account for $80 \%$ of hypercalcemia in patients with cancer. This protein shares a substantial sequence homology with $\mathrm{PTH}$; in fact, 8 of the first 13 amino acids at the N-terminal portion of PTH were identical. ${ }^{8} \mathrm{PTHrP}$ has multiple isoforms (PTHrP 141, PTHrP 139, and PTHrP 173). Like PTH, it enhances renal tubular reabsorption of calcium while increasing urinary phosphorus excretion. ${ }^{7}$ The result is both hypercalcemia and hypophosphatemia. However, unlike PTH, PTHrP does not increase $1,25(\mathrm{OH}) 2 \mathrm{D}$ and thus does not increase intestinal absorption of calcium and phosphorus. PTHrP acts on osteoblasts, leading to enhanced synthesis of receptor activator of nuclear factor- $\kappa B$ ligand (RANKL). ${ }^{7}$

In one study, PTHrP was detected immunohistochemically in prostate cancer cells. Iwamura and colleagues used 33 radical prostatectomy specimens from patients with clinically localized carcinoma of the prostate. ${ }^{9}$ None of these patients demonstrated hypercalcemia prior to the surgery. Using a mouse monoclonal antibody to an amino acid fragment, all cases demonstrated some degree of immunoreactivity throughout the cytoplasm of the tumor cells, but immunostaining was absent from inflammatory and stromal cells. ${ }^{9}$ 
FIGURE 3 Technetium-99m-Methylene Diphosphonate Bone Scintigraphy
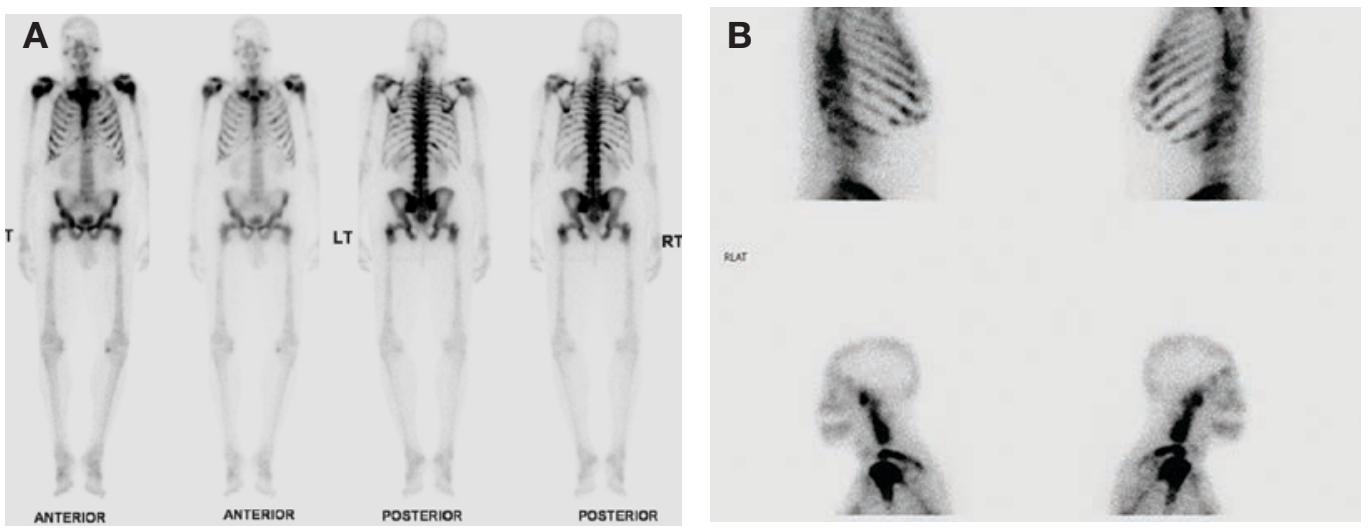

Scintigraphy shows increased bone radiotracer uptake in multiple bones consistent with metastases. A, Anterior and posterior views show increased uptake in shoulder joints, sternoclavicular joints, sternum, anterior and posterior ribs, cervical, thoracic, and lumbar spines, sacrum, pelvis, and hip joints (the femoral head/neck and intertrochanteric regions). B, Lateral view shows several foci of uptake involving the mandible and skull in the region of the temporomandibular joint bilaterally.

Furthermore, the intensity of the staining appeared to directly correlate with increasing tumor grade. ${ }^{9}$

Another study by Iwamura and colleagues suggested that PTHrP may play a significant role in the growth of prostate cancer by acting locally in an autocrine fashion. ${ }^{10}$ In this study, all prostate cancer cell lines from different sources expressed PTHrP immunoreactivity as well as evidence of DNA synthesis, the latter being measured by thymidine incorporation assay. Moreover, when these cells were incubated with various concentrations of mouse monoclonal antibody directed to PTHrP fragment, PTHrP-induced DNA synthesis was inhibited in a dose-dependent manner and almost completely neutralized at a specific concentration. Interestingly, the study demonstrated that cancer cell line derived from bone metastatic lesions secreted significantly greater amounts of PTHrP than did the cell line derived from the metastasis in the brain or in the lymph node. These findings suggest that PTHrP production may confer some advantage on the ability of prostate cancer cells to grow in bone. ${ }^{10}$

Ando and colleagues reported that neuroendocrine dedifferentiated prostate cancer can develop as a result of long-term ADT even after several years of therapy and has the potential to worsen and develop severe hypercalcemia. ${ }^{8}$ Neuron-specific enolase was used as the specific marker for the neuroendocrine cell, which suggested that the prostate cancer cell derived from the neuroendocrine cell might synthesize PTHrP and be responsible for the observed hypercalcemia. ${ }^{8}$

Other mechanisms cited for hypercalcemia of malignancy include other humoral factors associated with increased remodeling and comprise interleukin 1, 3, 6 (IL-1, IL-3, IL-6); tumor necrosis factor $\alpha$; transforming growth factor A and B observed in metastatic bone lesions in breast cancer; lymphotoxin; E series prostaglandins; and macrophage inflammatory protein $1 \alpha$ seen in MM.

Local osteolytic hypercalcemia accounts for about $20 \%$ of cases and is usually associated with extensive bone metastases. It is most commonly seen in MM and metastatic breast cancer and less commonly in leukemia. The proposed mechanism is thought to be because of the release of local cytokines from the tumor, resulting in excess osteoclast activation and enhanced bone resorption often through RANK/RANKL interaction.

Extrarenal production of $1,25(\mathrm{OH}) 2 \mathrm{D}$ by the tumor accounts for about $1 \%$ of cases of hypercalcemia in malignancy. $1,25(\mathrm{OH}) 2 \mathrm{D}$ causes increased intestinal absorption of calcium and enhances osteolytic bone resorption, resulting in increased serum calcium. This mechanism is most commonly seen with Hodgkin and non-Hodgkin lymphoma and had been reported in ovarian dysgerminoma. ${ }^{7}$

In our patient, bone imaging showed 
osteoblastic lesions, a finding that likely contrasts the local osteolytic bone destruction theory. PTHrP was not significantly elevated in the serum, and PTH levels ruled out any form of primary hyperparathyroidism. In addition, histopathology showed no evidence of mosaicism or neuroendocrine dedifferentiation.

Findings in aggregate tell us that an exact pathophysiologic mechanism leading to hypercalcemia in prostate cancer is still unclear and may involve an interplay between growth factors and possible osteolytic materials, yet it must be studied thoroughly.

\section{CONCLUSIONS}

Hypercalcemia in pure metastatic adenocarcinoma of prostate is a rare finding and is of uncertain significance. Some studies suggested a search for unusual histopathologies, including neuroendocrine cancer and neuroendocrine dedifferentiation..$^{8,11}$ However, in adenocarcinoma alone, it has an uncertain pathophysiology that needs to be further studied. Studies needed to investigate the role of PTHrP as a growth factor for both prostate cancer cells and development of hypercalcemia and possibly target-directed monoclonal antibody therapies may need to be extensively researched.

\section{Author disclosures}

The authors report no actual or potential conflicts of interest with regard to this article.

\section{Disclaimer}

The opinions expressed herein are those of the authors and do not necessarily reflect those of Federal Practitioner, Frontline
Medical Communications Inc., the US Government, or any of its agencies. This article may discuss unlabeled or investigational use of certain drugs. Please review the complete prescribing information for specific drugs or drug combinations -including indications, contraindications, warnings, and adverse effects-before administering pharmacologic therapy to patients.

\section{References}

1. Gastanaga VM, Schwartzberg LS, Jain RK, et al. Prevalence of hypercalcemia among cancer patients in the United States. Cancer Med. 2016;5(8):2091-2100. doi:10.1002/cam4.749

2. Grill V, Martin TJ. Hypercalcemia of malignancy. Rev Endocr Metab Disord. 2000;1(4):253-263. doi:10.1023/a:1026597816193

3. Halabi S, Kelly WK, Ma H, et al. Meta-analysis evaluating the impact of site of metastasis on overall survival in men with castration-resistant prostate cancer. J Clin Oncol. 2016;34(14):1652-1659. doi:10.1200/JCO.2015.65.7270

4. Chang SS. Overview of prostate-specific membrane antigen. Rev Urol. 2004;6(suppl 10):S13-S18.

5. Kummar S, Fogarasi M, Canova A, Mota A, Ciesielski T. Cytokeratin 7 and 20 staining for the diagnosis of lung and colorectal adenocarcinoma. $\mathrm{Br} J$ Cancer. 2002;86(12):1884-1887. doi:10.1038/sj.bjc.6600326

6. Avashia JH, Walsh TD, Thomas AJ Jr, Kaye M, Licata A. Metastatic carcinoma of the prostate with hypercalcemia [published correction appears in Cleve Clin J Med. 1991;58(3):284]. Cleve Clin J Med. 1990;57(7):636-638. doi:10.3949/ccjm.57.7.636

7. Goldner W. Cancer-related hypercalcemia. J Oncol Pract. 2016;12(5):426-432. doi:10.1200/JOP.2016.011155

8. Ando T, Watanabe K, Mizusawa T, Katagiri A. Hypercalcemia due to parathyroid hormone-related peptide secreted by neuroendocrine dedifferentiated prostate cancer. Urol Case Rep. 2018;22:67-69. doi:10.1016/.jeucr.2018.11.001

9. Iwamura M, di Sant'Agnese PA, Wu G, et al. Immunohistochemical localization of parathyroid hormonerelated protein in human prostate cancer. Cancer Res. 1993;53(8):1724-1726.

10. Iwamura M, Abrahamsson PA, Foss KA, Wu G, Cockett AT, Deftos LJ. Parathyroid hormone-related protein: a potential autocrine growth regulator in human prostate cancer cell lines. Urology. 1994;43(5):675-679. doi:10.1016/0090-4295(94)90183-x

11. Smith DC, Tucker JA, Trump DL. Hypercalcemia and neuroendocrine carcinoma of the prostate: a report of three cases and a review of the literature. J Clin Oncol. 1992;10(3):499-505. doi:10.1200/JCO.1992.10.3.499 